\title{
Enseñanza de autocontrol \\ de la biopelícula dental a gestantes, en hospitales públicos de Paraguay
}

Rossana Sotomayor Ortellado ${ }^{1}$; Graciela Velazquez de Saldivar ${ }^{2}$;

Myriam Agüero ${ }^{3}$

\section{Resumen}

El objetivo del presente estudio fue evaluar la efectividad de estrategias de enseñanza de autocontrol del biofilme dental, en embarazadas atendidas en hospitales públicos del Departamento Central de Paraguay. Materiales y Métodos: Este estudio, de tres meses de duración, contó con una muestra de 38 embarazadas: GE-Grupo experimental ( $\mathrm{n}=19)$ y GC-Grupo control ( $n=19)$. En el primer encuentro, denominado en adelante Basal (B), se realizaron las invitaciones, seguida de la firma del consentimiento informado; Intervención para el GE: Llenado de un formulario de conocimientos, prácticas y autoeficacia percibida; evaluación de la higiene, utilizando el índice de O'Leary, higiene bucal orientada; charla educativa de 10 minutos, con rotafolio ilustrado, a un máximo de 4 participantes a la vez; ficha clínica; profilaxis e inactivación de lesiones de caries según necesidad. Para el GC se realizaron los mismos procedimientos, solo que el contenido educativo fue transmitido exclusivamente de forma verbal. La segunda medición tuvo lugar en una cita control (Cc) a los 7,14 o 21 días (según disponibilidad de la gestante). Resultados: Hubo una mayor remoción del biofilme en el GE en la Cc: En B, el índice de O'Leary fue de $88 \%$ para el GE, y $85 \%$ para el GC, y en la Cc, $14 \%$ y $58 \%$, respectivamente $(p=0,00001)$. Los conocimientos: En B el 89\% de los participantes (17) tuvo menos del $60 \%$ de respuestas correctas; en la Cc el 58\% (11) del GE, y 16\% (3) del GC ( $p=0,007)$ tuvieron $60 \%$ o más respuestas correctas. Las prácticas y la autoeficacia percibida no tuvieron variaciones significativas. Conclusión: Las estrategias seleccionadas se mostraron eficaces para mejorar el autocontrol del biofilme dental en la población estudiada.

Palabras Clave: Mujeres embarazadas, educación, placa dental, higiene bucal.

\footnotetext{
1. Master en Odontología con énfasis en Odontopediatría, Universidad Autónoma del Paraguay. Encargada de la Cátedra de Odontopediatría de la Universidad Nacional de Concepción, Concepción, Paraguay.

2. Doctora en Bioquímica, Directora de Investigación de la Universidad del Cono Sur de las Américas, Universidad Autónoma del Paraguay, Asunción, Paraguay.

3. Especialista en Odontopediatría, Universidad Autónoma del Paraguay, Asunción, Paraguay.
} 
Artigo Original

\section{Ensino do auto-controle do biofilme dental a gestantes em hospitais públicos do Paraguai}

\section{Resumo}

O objetivo do presente estudo foi avaliar a efetividade de estratégias de ensino de autocontrole de biofilme dentário em grávidas que procuraram hospitais públicos no departamento central do Paraguai. Material e Métodos: Este estudo clínico de três meses de duração foi composto por uma amostra de 38 grávidas: GE- grupo experimental $(n=19)$ e GC- grupo controle $(n=19)$. No primeiro encontro, denominado baseline (B), foram realizados os convites, seguido pela assinatura de consentimento informado. A Intervenção no GE consistiu em: Formulário de conhecimentos, prácticas e auto eficácia percebida; índice de O'leary, seguido de higiene bucal orientada; palestra educativa de 10 minutos de duração, utilizando um flip chart ilustrado com até 4 participantes por vez; profilaxia e inativação de lesões de cárie segundo a necessidade. No GC, os mesmos procedimentos foram realizados; entretanto, o conteúdo educacional foi transmitido apenas verbalmente. A segunda medição foi realizadaem uma data de controle (Cc) que podia ocorrer aos 7, 14 ou 21 dias após a primeira consulta (dependendo da disponibilidade da gestante). Resultados: Houve maior remoção de biofilme no GE em comparação ao GC em Cc (B- índice de O'Leary GE: 88\% e GC: $85 \%$ / Cc? ( $14 \%$ e $58 \%$ respectivamente; $p=0,00001$ ). Os conhecimentos: em B, 89\% dos participantes (17) tiveram menos de $60 \%$ de respostas corretas, já em Cc, $58 \%$ (11) de GE e $16 \%$ (3) de GC ( $\mathrm{p}=0,007$ ) tiveram $60 \%$ ou mais respostas correctas. As práticas e auto-eficácia não tiverem variação significativa. Conclusão: As estratégias empregadas mostraram-se eficazes para melhorar o controle do biofilme dentário em gestantess.

Palavras chave: Mulheres grávidas, educação, placa dental, higiene bucal.

Original article

\section{Teaching of self-monitoring dental biofilm to pregnant women, in public hospitals of Paraguay}

\section{Abstract}

The aim of this study was to evaluate the effectiveness of selected strategies for teaching self-control of dental biofilm in pregnant women attending public hospitals from the Central
Department of Paraguay. This study, of three months of duration had, a sample of 38 pregnant women (19 per group): experimental (GE) and control (GC). In Basal (B), invitations were carried out to participate (person to person), followed by signing of the informed consent document. The 
intervention, for the GE, consisted of: Form to determine knowledge, practices and self-efficacy; educational presentation of 10 minutes duration with illustrated flipchart (to a maximum of 4 participants); O'leary index; practice of oral hygiene with individual guidance; Clinical inspection and dental record; Dental prophylaxis and inactivation of carious lesions in cases that were indicated. For the GC: The same procedures, only that the educational content was deliberated exclusively verbally. Each participant could return at 7, 15 or 21 days for a control appointment (Cc) depending on time availability. Results: The rate

\section{Introducción}

En el período gestacional ocurren cambios en la cavidad bucal, tales como descenso del $\mathrm{pH}$ y disminución de la capacidad buffer de la saliva, así como el aumento en la vascularización y flujo sanguíneo del tejido periodontal. Estas variaciones han sido relacionadas con efectos adversos, reversibles e irreversibles, en la encía o en los dientes. La prevalencia de dichos efectos es mayor cuando las gestantes presentan alteraciones gingivales y/o caries antes del embarazo. Cabe destacar que la mayoría de los efectos negativos que estos cambios suscitan, pueden ser controlados con una buena higiene bucal. ${ }^{1,2}$

Al revisar los antecedentes existentes en Paraguay con relación a la salud de gestantes, se encontraron estudios transversales que exponen datos de prevalencia de biofilme dental, caries y gingivitis, así como, encuestas de conocimientos, actitudes y prácticas de salud e higiene bucal. En ellos se puede apreciar lo mucho que se puede realizar en términos de prevención en esta población. Entre los trabajos consultados of O'leary index improve for GE, in B was $88 \%$ for GE and $85 \%$ for GC, and in Cc, $14 \%$ and $58 \%$ respectively ( $p=0.00001)$. Knowledge: In B $89 \%$ of participants (17) achieved $<60 \%$ correct responses, in Cc $=$ or $>60 \%$ correct answers: $58 \%$ (11) of GE and $16 \%$ (3) of GC ( $\mathrm{p}=0.007)$. The practices and perceived self-efficacy did not have significant variation. Conclusion: The selected strategies to teach were effective to improve self-control of dental biofilm in the study population.

Key words: Pregnant woman, education, dental plaque, oral hygiene.

está el desarrollado en el Hospital Materno-Infantil de Santísima Trinidad, con una muestra de 128 mujeres, donde se encontró una prevalencia de $91 \%$ de caries y $80 \%$ de gingivitis, con un CPOD promedio de $10.7 .^{3}$

En otro, realizado en la ciudad de Luque, con una muestra de 142 embarazadas de 13 -42 años de edad, se encontró que el nivel de conocimientos de salud bucal en aquellas mujeres que solo alcanzaron el nivel primario de educación, fue desfavorable; en tanto que las prácticas de higiene bucal fueron desfavorables para aquellas que alcanzaron el nivel primario y/o el nivel secundario. $^{4}$

Otro estudio, llevado a cabo en 148 adolescentes embarazadas que realizaron sus controles prenatales en un hospital de San Lorenzo, se encontró que dichas adolescentes tenían un nivel medio-bajo en conocimiento sobre prevención y un nivel de prácticas desfavorable, con una media de presencia de biofilme dental de $94 \%$; gingivitis en el 79\%, y valores promedio de CPOD de 6 a $11 .^{5}$ 
Si bien el Ministerio de Salud Pública y Bienestar Social (MSPBS) de Paraguay promueve la realización de al menos un examen odontológico durante la gravidez, como se constata en su Guía para la salud integral de la niñez-adolescencia y madre embarazada, del año 2011, en un estudio retrospectivo, de una importante región del país, se observó que las consultas de control que realizaban las embarazadas disminuyeron en un 60\% (de 11.258 a 4.554) del año 2008 al año $2011 .^{6}$

\section{Materiales y métodos}

Se realizó un ensayo clínico de tres meses de duración. Se efectuaron mediciones en Basal (B) y en una Cita control (Cc) (se le dio tres oportunidades a cada participante para asistir a la Cc) desde los 7, 14 y 21 días, luego de B. El estudio fue aprobado por el Comité de ética de la Universidad Autónoma del Paraguay. Fueron invitadas a participar del estudio un total de 64 embarazadas que asistían a su control ginecológico en tres hospitales públicos materno-infantiles del Departamento Central de Paraguay. Los hospitales en cuestión fueron tres, de un total de cinco con atención dirigida a mujeres embarazadas y niños, en el espacio geográfico referido. Para el cálculo del tamaño de la muestra se realizó un estudio preliminar en 5 pacientes del Grupo control, donde se obtuvo el siguiente dato: Índice de O'Leary de $85 \pm 18$. Dos preguntas fueron reformuladas después de esta prueba para mejorar la interpretación de las mismas. Esperando una diferencia de media de 15 (en el Índice de O’Leary) y un desvío estándar de 18, entre el Grupo experimental y el Grupo control, el tamaño de la muestra debió ser de 19 individuos, como mínimo en cada grupo, para un alfa bilateral $=0,10$, Beta $=0,20$ (tamaño de muestra requerido para comparar medias empleando la prueba $\mathrm{t}$ ).

Participaron del primer encuentro (B o día de la intervención) 60 gestantes que firmaron el consentimiento informado, luego de recibir información sobre el estudio. Dos mujeres no aceptaron participar del estudio, alegando falta de tiempo. Además, fueron excluidas 2 mujeres; una con prótesis completa en una de sus arcadas, y la otra, portadora de aparatología de ortodoncia.

Las participantes no tenían conocimiento de a qué grupo pertenecían, si al Grupo experimental o al Grupo control. Se realizaron las intervenciones en dos hospitales: San Pablo y Santísima Trinidad, y en Villa Elisa, los controles. Todas las participantes recibieron mensajes de texto vía celular, como recordatorio y motivación a asistir a la Cc. Concurrieron a la Cc 19 del Grupo experimental y 16 del Grupo control en el mismo lapso de tiempo. Luego, se igualó el número de mujeres de cada grupo (GE y GC). Solo las participantes que retornaron a la Cc fueron incluidas en los análisis estadísticos.

\section{Intervención: Componentes:}

1) Formulario para determinar conocimientos, actitudes y prácticas, que incluyó 29 preguntas (3 abiertas y 26 cerradas). Temas explorados: Datos demográficos, Biofilme dental o placa bacteriana ¿qué es?; Autohigiene oral: ¿cómo aprendió?, frecuencia con la que se realiza el cepillado y uso de hilo dental; confianza y seguridad en la realización del cepillado de dientes; caries y gingi- 
vitis: ¿qué son?, reconocimiento de signos y síntomas; consulta odontológica durante el embarazo: barreras; consumo de azúcar durante el embarazo: percepción de aumento del consumo, número de veces que ingiere azúcar por día; creencias fatalistas sobre la salud bucal durante el embarazo y la caries durante la infancia; cuidado de la salud bucal del bebé.

2) Implementación de estrategias educativas, consistentes en:

2.1) Charla sobre el biofilme dental (su formación, sus consecuencias y cómo controlarlo), higiene bucal y consumo moderado de azúcar. Duración: 10 minutos. Para el grupo experimental (GE) la charla fue grupal (con un límite de 4 participantes) realizada por una especialista que utilizó un rotafolio impreso en papel fotográfico mate de $250 \mathrm{~g}$. Tamaño A3 a full color y un macromodelo de la boca con un cepillo de dientes y un espejo facial para mostrarle la tinción.

Se les entregaron kits de higiene bucal a todas las participantes: Cepillo de dientes (Colgate ${ }^{\circledR}$ Classic $\left.{ }^{\circledR}\right)$ de cerdas suaves, pasta dental (Colgate ${ }^{\circledR}$ Máxima protección anticaries), reloj de arena de 2 minutos.

2.2) La tinción del biofilme se hizo con una solución de colorante azul y colorante rojo (Replak, Dentsply, Rio de Janeiro, Brasil), y se colocó una gota en un cotonette por arcada. Se utilizó el índice de O'Leary: Se dividió la cavidad bucal en cuatro cuadrantes I, II, III y IV (hemiarcada superior e inferior derecha e izquierda). Caras dentarias valoradas: Vestibular, Palatino, Mesial y Distal de todas las piezas dentarias naturales presentes; No se discriminó la extensión, la zona de depósito por superficie ni el grado de maduración del biofilme. No se registraron piezas que presentaron tinción leve a nivel de la unión dento-gingival. No se registraron superficies con destrucción coronaria amplia a nivel de la cervical. Las examinadoras fueron capacitadas durante 3 años en el uso del índice empleado, y efectuaron un entrenamiento de concordancia previo al estudio, haciendo uso de un documento disponible en Power Point (Slide Share. Murrieta J). Las tinciones se hicieron en horarios considerados próximos a uno de los horarios habituales de higiene de las participantes: 7.30 a $8.30 \mathrm{hs;}$ y 13.00 a 14.00hs. Seguidamente se realizaron:

2.3) Enseñanza activa de técnica de cepillado, en una sala dotada de lavamanos. Se les pidió a las gestantes que observen la/s superficie/s teñida/s en un espejo, y se les orientó, relacionando lo observado con la información facilitada en la charla.

Los temas abarcados en la misma fueron: Forma correcta de cepillarse, dividiendo la boca en cuadrantes y dedicando aproximadamente 30 segundos a cada cuadrante: tiempo total, dos minutos (en este momento se destacó el uso del reloj de arena como referencia del tiempo de cepillado), y se destacó la necesidad de incluir la lengua en la higiene; Pasta dental con flúor, tres veces al día, y Uso del hilo dental.

Se solicitó que inicien el cepillado de forma habitual para verificar la técnica utilizada, y a continuación se mostraron posiciones y movimientos a corregir con la ayuda del macromodelo; la posición expuesta: una angulación de alrededor de 45 grados en caras 
libres. En cuanto a movimientos se les brindó la opción de intentar la combinación de movimientos de vibración y barrido hacia el incisal, o movimientos circulares. Los movimientos de vaivén se limitaron a las superficies masticatorias. Igualmente se mostró la necesidad de uso de hilo dental en las superficies interdentales (pasando el hilo en una sola superficie interdental). A continuación se alentó a practicar los nuevos movimientos con el cepillo de dientes. Duración aproximada: 5 minutos.

3) Inspección clínica y adecuación del medio bucal, en el sillón odontológico facilitado por cada hospital, haciendo uso de la luz del equipo y espejo bucal. La actividad consistió en: a) Profilaxis con cepillo de Robinson y pasta para profilaxis. Con la boca libre de biofilme dental se confeccionó el odontograma. Las lesiones de caries fueron evaluadas por superficie, y se consideraron como tales la pérdida de tejido en esmalte y dentina; superficies con obturaciones provisorias con materiales como óxido de zinc y eugenol. Fueron consideradas manchas blancas aquellas localizadas en zonas de retención de placa. b) Adecuación del medio bucal: Remoción de sarro, inactivación de lesiones de caries utilizando pastas a base de óxido de zinc libre de eugenol para el sellado provisorio; pulido de obturaciones desbordantes, indicaciones de la necesidad de extracción y terapia pulpar, especificando días y horarios para estos tratamientos con el staff de profesionales de los hospitales.

Para el G0rupo control (GC), la charla en B fue individual y exclusivamente verbal (procedimiento habitual en los hospitales donde se desarrolló el estudio). Se les entregó cepillo de dientes y pasta dental (idénticas carac- terísticas a lo proveído al GE). Se aplicaron los mismos formularios y realizaron los mismos procedimientos clínicos efectuados con el GE en las dos citas.

Controles: Cc: Índice de O'Leary, y Formulario 2, que constó de 10 preguntas iguales o similares a las del formulario utilizado en B sobre los temas centrales, como: conocimientos sobre biofilme dental y sus consecuencias, además de higiene y consumo de azúcar, todo ello seguido de refuerzo de las debilidades; Charla sobre el cuidado de la salud bucal del bebé; Entrega de obsequios para el bebé: toallitas y pañales.

\section{Recursos humanos empleados:}

Un total de 5 profesionales participaron de la colecta de datos. En B para el GE, 3 profesionales; para el GC, 2 profesionales, y en la Cc, 2 profesionales para ambos grupos.

Las observaciones para el registro de superficies teñidas y odontograma, así como las charlas educativas estuvieron a cargo de solo 2 profesionales, con experiencia clínica de 8 y 10 años, ambas especializadas en Odontopediatría; una de forma exclusiva para el GE, y la otra para el GC.

Antes y después de la intervención: El índice de O'Leary fue calculado de forma individual para cada participante utilizando la fórmula:

Índice de placa $=\frac{\text { Superficies teñidas }}{\text { Dientes presentes x } 4} \times 100$

Para cada grupo (GE y GC) se determinó el promedio en B y Cc. 


\begin{tabular}{|c|c|c|c|c|}
\hline Grupo & N & $\begin{array}{c}\text { Basal \% } \\
\text { promedio; DS }\end{array}$ & $\begin{array}{c}\text { Post interv. \% } \\
\text { promedio; DS }\end{array}$ & Valor p \\
\hline Experimental & 19 & $88,29 \pm 13,31^{\star}$ & $14,29 \pm 7,72^{\star *}$ & \multirow{2}{*}{$<0,00001^{\star *}$} \\
\hline Control & 19 & $85,00 \pm 17,36$ & $58,66 \pm 20,53$ & \\
\hline
\end{tabular}

*NS; **Prueba Mann-Whitney.

Cuadro 1. Comparación Índice de O'Leary antes y después de la intervención en ambos grupos (experimental y control).

\section{Conocimiento, Prácticas}

y Percepción de auto eficacia:

Se clasificaron las respuestas teniendo en cuenta si cada participante alcanzó o superó el 60\%, o no alcanzó el 60\%. Ver cuadro.

\section{Resultados}

Con relación al Índice de O'Leary, en Basal alcanzaron promedios similares: 88,29\% GE, y 85,00\% GC. En tanto, en la cita posterior a la intervención se observa: El GE tuvo en promedio 14,29\%, mientras que el grupo control, 58,66\% Al comparar los promedios alcanzados antes y después de la inter- vención se verificó una diferencia estadísticamente significativa entre el Grupo experimental y el Grupo de control, con un valor $p<0,00001$; Prueba Mann Whitney. (Cuadro 1 y Gráfico 1)

En cuanto al Conocimiento, fue homogéneo en Basal; en ambos grupos 17 gestantes, lo que equivale a decir que el $89 \%$ de la muestra no logró el $60 \%$ de respuestas correctas: Conocimiento no favorable.

Luego de la intervención, 11 (58\%) del GE lograron igualar o superar el $60 \%$ de respuestas correctas, y en el GC, 3 (16\%); esto se mostró estadísticamente significativo (Valor $\mathrm{p}=0,007$; Prueba Chi 2). Ver Gráfico 2.
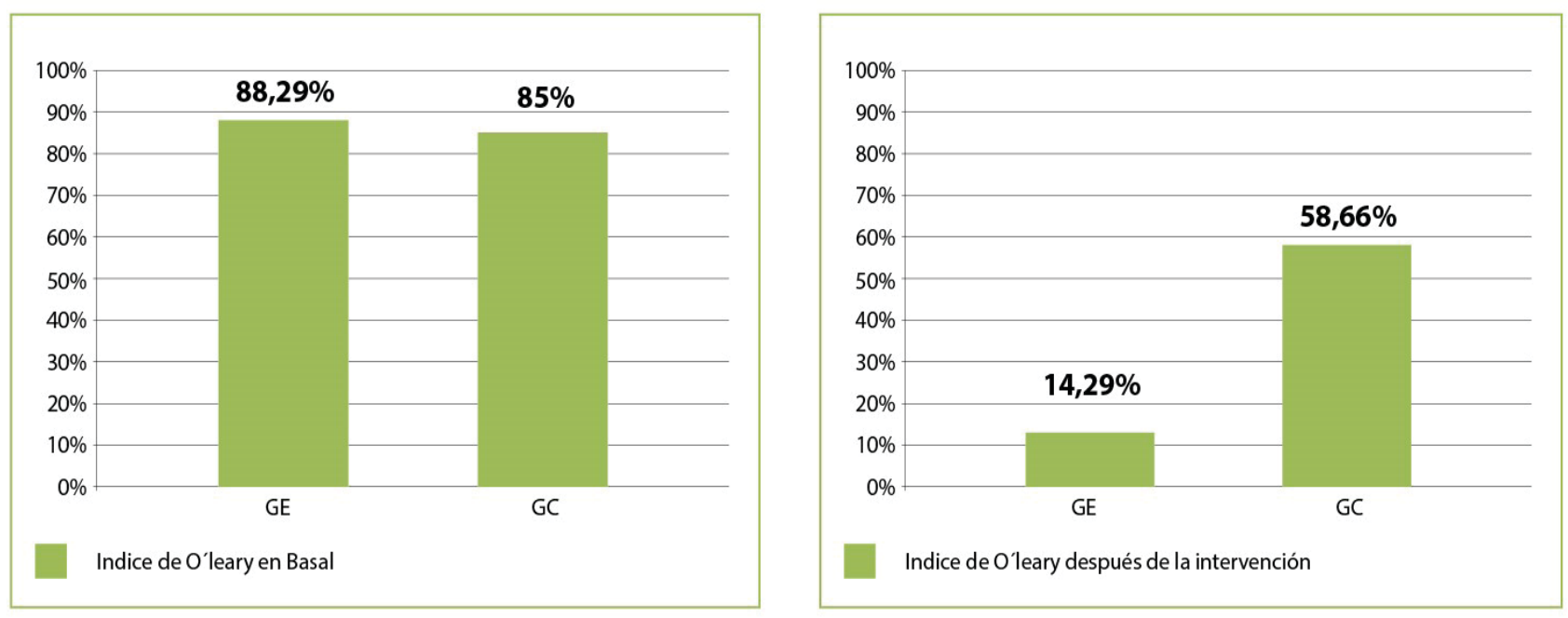

Gráfico 1. Comparación de valores obtenidos con el Índice de O'Leary antes y después de la intervención para ambos grupos (Experimental $n=19$; Control $n=19$ ). 

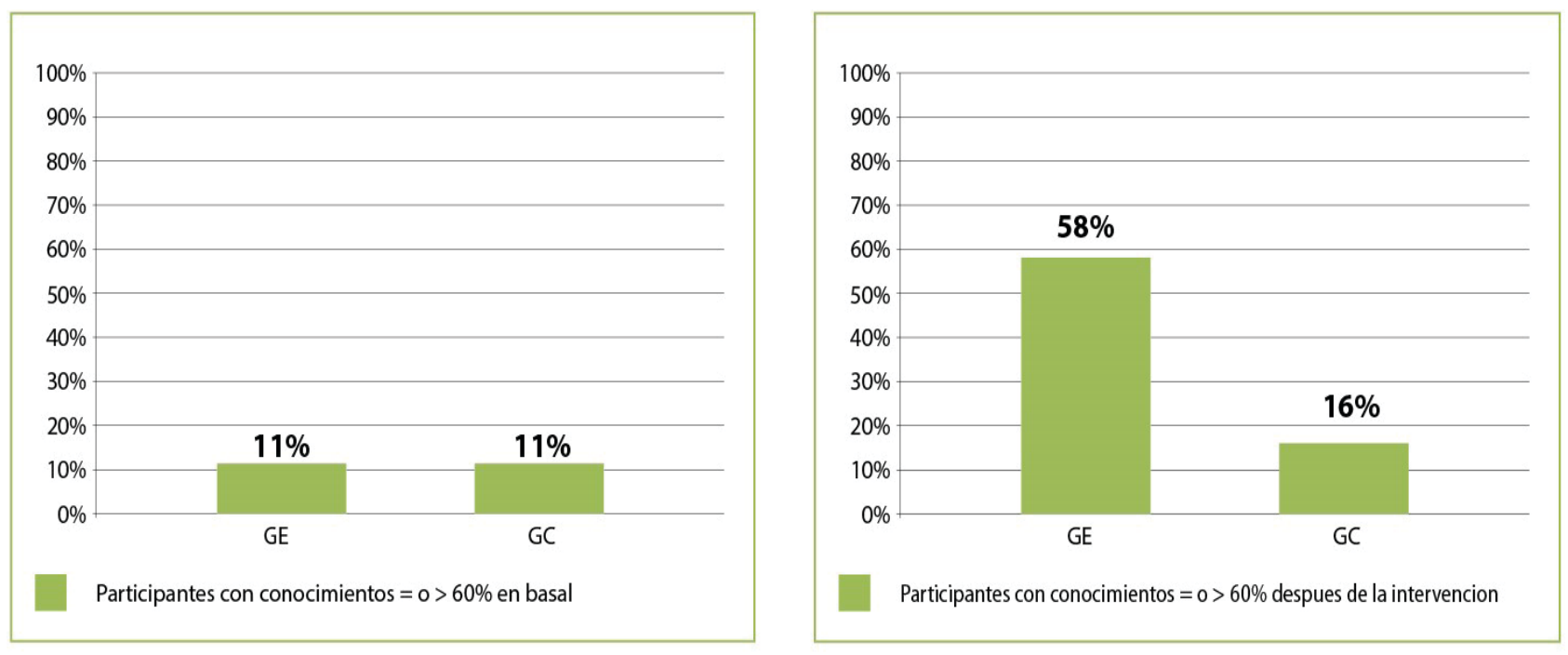

Gráfico 2. Comparación entre grupos del porcentaje de embarazadas que mostraron un nivel de Conocimiento $=0>60 \%$ antes $y$ después de la intervención. (Experimental $n=19$; Control $n=19$ ).

En las preguntas relacionadas con las Prácticas, las mayores frecuencias en Basal, para ambos grupos, se verificaron en aquellas que alcanzaron o superaron en $60 \%$ de respuestas favorables: 11 (58\%) en GE y en GC. Luego de la intervención esta situación se dio en 17 (89\%) del GE, y 13 (68\%) del GC; es decir, la diferencia al comparar el antes y después no fue significativa (Valor $\mathrm{p}>$ 0,05). Resultados se presentan en el Gráfico 3.

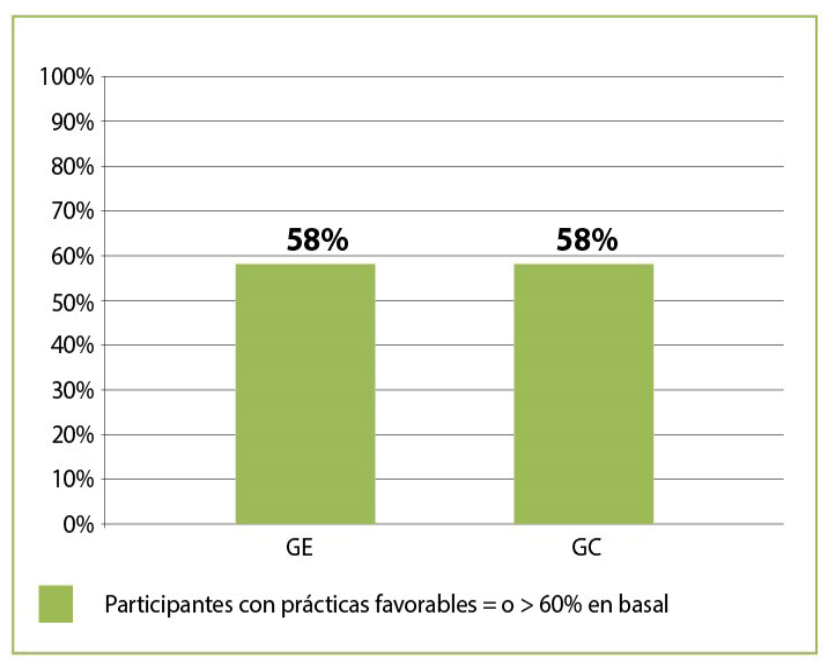

Con relación a la confianza para realizar el cepillado de dientes, Autoeficacia percibida, se tiene el siguiente resultado: Ya en Basal hubo mayores frecuencias para la respuesta: "sí, considero que me cepillo bien los dientes": 11 (58\%) y 12 (63\%) para los grupos Experimental y Control, respectivamente. En la cita posterior a la intervención, hubo un ligero aumento en el número de participantes que dieron esta respuesta; en el

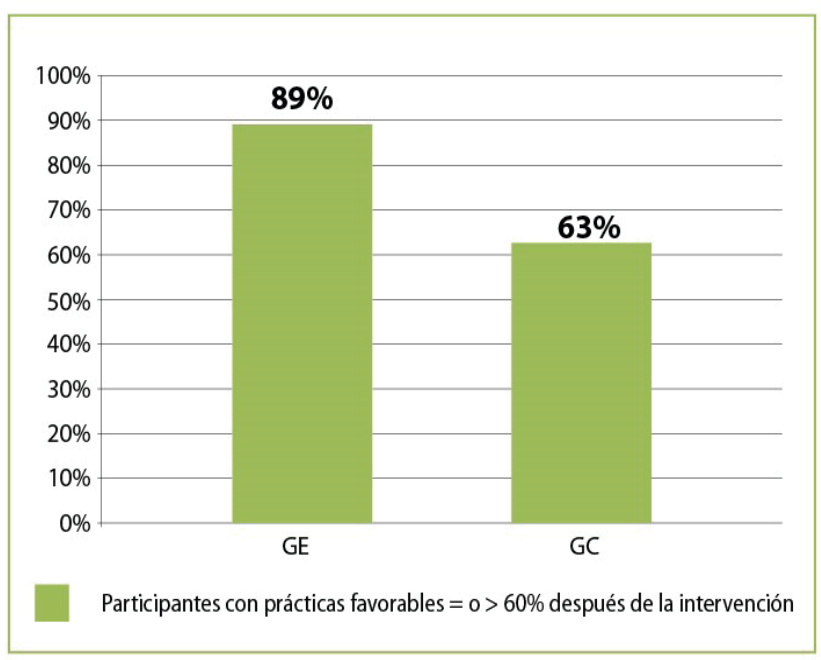

Gráfico 3. Comparación entre grupos del porcentaje de embarazadas que manifestaron realizar prácticas favorables para la salud bucal $=0>60 \%$ antes y después de la intervención. (Experimental $n=19$; Control $n=19$ ). 

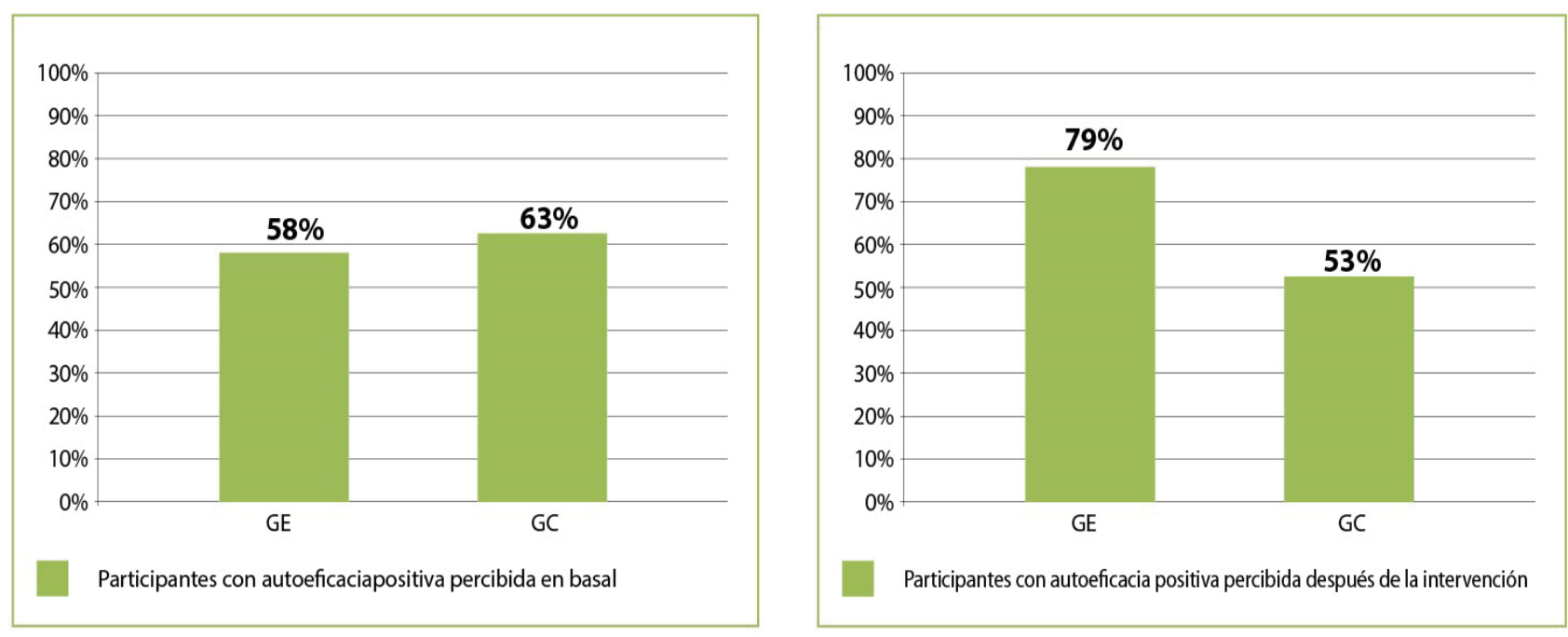

Gráfico 4. Comparación entre grupos del porcentaje de embarazadas con autoeficacia percibida positiva, antes y después de la intervención. (Experimental $n=19$; Control $n=19$ ).

GE, 15 (79\%), mientras que en el GC 10 (53\%). La diferencia entre el antes y después no fue significativa (Valor p>0,05). Gráfico 4.

\section{Discusión}

La literatura científica justifica la necesidad de elaborar programas educativos sobre salud bucal para embarazadas. Una de las principales fundamentaciones de ello es el rol clave que cumplen las mujeres en el seno familiar y su gran influencia sobre factores determinantes en la salud como dieta e higiene. Así también, la oportunidad que representa el momento de la gestación, por el mayor acercamiento a los profesionales del área de la salud. ${ }^{11,12}$ La tarea pendiente sería cómo plantear estos programas educativos.

Recordando que con la versión utilizada del Índice de O'Leary para este estudio, los porcentajes elevados indican mayor presencia de biofilme; los promedios en Basal fueron considerablemente elevados: $88 \%$ para el Grupo experimental, y 85\% para el Grupo control, y siendo significativo el descenso posterior a la intervención para el Grupo experimental, que en promedio tuvo un $14 \%$ frente al $58 \%$ obtenido por el Grupo control. Similar resultado obtuvo Bonilla et al (2008) ${ }^{7}$ El mejor control del biofilme, significativo, en el Grupo experimental, puede deberse a la sinergia del conjunto de estrategias educativas seleccionadas. Ahora bien, se tiene en cuenta que el Grupo control también verificó una disminución del biofilme en la cita control, podrían haber otros factores a ser explorados, tales como el impacto de salir de la rutina en hospitales públicos. En lo que respecta a limitaciones del estudio, cabe destacar que el tiempo de seguimiento fue breve, por lo que no se pueden garantizar efectos positivos a largo plazo.

El conocimiento también mejoró de forma significativa en el Grupo experimental, que luego de la intervención logró un promedio de 58\% de participantes con respuestas correctas, igual o superior al $60 \%$, frente al Grupo control, con un $16 \%$. Ambos grupos, en el formulario aplicado en Basal, obtuvieron un promedio de $89 \%$ de participantes con repuestas correctas inferior 
al $60 \%$, esto también dio una diferencia significativa $(\mathrm{p}=0,007)$. Esto ocurrió de igual manera en los estudios de Bonilla et al 2008; Louis Lin et al, 20115,6. Sin embargo, las prácticas (Número de veces que se cepillan los dientes por día; Uso diario del hilo dental; Consumo de azúcar) verificadas en la encuesta no mejoraron de forma significativa después de la intervención, al promediar los resultados individuales, aunque el promedio de participantes con respuestas favorables fue ligeramente mayor para el Grupo experimental, $89 \%$, frente a $68 \%$ para el Grupo control. Esto destaca que no siempre lo que se manifiesta en una encuesta guarda relación estricta con lo que se practica, ya que los cambios que se observaron en la práctica fueron cualitativos y no cuantitativos. Entonces, la ya conocida pero no siempre utilizada práctica de higiene bucal, realizada por la paciente en consultorio, "observada y orientada" por un profesional, podría ser una herramienta importante en la educación bucal de embarazadas en estos hospitales.

Una variable a menudo mencionada en la literatura como de gran influencia en el desempeño de hábitos de higiene es la autoeficacia percibida (Buglar et al 2010; Clarkson et al 2009)7,8 En los estudios citados se puede apreciar un aumento de la autoeficacia, y los autores la señalan como un buen predictor para determinar la adherencia o no a una práctica en particular.

No obstante, en el presente estudio ambos grupos de la muestra expresan confianza en ejecutar el cepillado de dientes desde Basal; $58 \%$ de las participantes del Grupo experimental y 63\% del Grupo control. En tanto, la encuesta de seguimiento también reflejó un aumento, aunque mayor en el Grupo experimental, con 79\% de participantes con autoeficacia percibida de forma positiva; no obstante, el mismo no fue estadísticamente significativo frente al $53 \%$ de participantes que se sentía confiado en el Grupo control, respecto a su manera de efectuar el cepillado dental.

La disminución de la confianza en la práctica en el Grupo control pudiera darse por la confusión que genera el hecho de que el mensaje al respecto sea solo dicho y no mostrado ni experimentado.

Finalmente se puede afirmar que las estrategias escogidas tuvieron un balance positivo en el logro del objetivo.

\section{Conclusiones}

En la población estudiada, las características demográficas y clínicas más destacadas fueron que la mayoría, al promediar ambos grupos, tenía más de 20 años de edad, y manifestaron haber alcanzado el nivel secundario de instrucción académica, así como vivir en uniones no constituidas de forma legal. Igualmente, también se observó que en la inspección presentaron al menos una lesión de caries u obturación provisoria, al igual que signos de gingivitis, y entre 1 y 4 piezas dentarias ausentes.

Las estrategias seleccionadas se mostraron eficaces para:

- Mejorar el control del biofilme en los controles efectuados entre los días 7 y 21, para una significativa mayoría del grupo experimental.

- Aumentar el conocimiento de forma significativa en el Grupo experimental.

En tanto que no fue eficaz para:

- Aumentar en forma significativa las prácticas verificadas solo a través del formulario (las manifestadas por las participantes). 


\section{Agradecimientos}

A los responsables de los hospitales maternos
infantiles: Santísima Trinidad, San Pablo y Villa
Elisa que brindaron el espacio para la realiza- ción de este estudio y a los funcionarios de las instituciones citadas, que facilitaron el trabajo. A las doctoras: Celia Marmol, Marythe Campuzano, Karen Gibons y Sandra Martinez por la colaboración en los días de recolección de datos.

\section{Referencias bibliográficas}

1. Chamilco A. Soriano A. Variación del PH y flujo salival durante el periodo gestacional en embarazadas de un servicio asistencial público. [Tesis]. Lima (Perú): Universidad Nacional Mayor de San Marcos. Consultado en fecha 10 de diciembre del 2013 [internet] Disponible en: http://cybertesis.unmsm.edu.pe/handle/cybertesis/3372.

2. Laine MA. Effect of pregnancy on periodontal and dental health; Acta Odontol Scand. [internet] 2002 Oct; 60(5): 257-64.

3. Grutter A. Nivel de conocimiento y práctica en salud bucodental, condición bucal en embarazadas del Hospital Materno Infantil de Santísima Trinidad. [Tesis]. Asunción (Paraguay): Universidad Autónoma del Paraguay, Facultad de Odontología Pierre Fauchard; 2011.

4. Medina A. Cabañas A. Jacquett N. Nivel de conocimiento, actitud y práctica sobre salud bucodental en mujeres embarazadas de un hospital público de la ciudad de Luque en el año 2011. [Tesis]. Asunción (Paraguay): Universidad Autónoma del Paraguay, Facultad de Odontología Pierre Fauchard; 2012. 120p.

5. Cabrera R; Chirife T, Jacquett N. Condición Oral y Nivel de Conocimiento y Práctica sobre prevención en salud bucal de adolescentes embarazadas en un hospital público de San Lorenzo en el año 2007. [Tesis]. Asunción (Paraguay): Universidad Autónoma del Paraguay, Facultad de Odontología Pierre Fauchard; 2008.

6. Mongelós de Idoyaga MG, Perdomo M. Impacto de la gratuidad en el acceso a servicios odontológicos, $2008-2011$. Paraguay oral research. [Internet]. 2013. [27.08.12] 2 (1): 17-22 Disponible en: http://www.paraguayoral.com.py/revista/ a2v1/ART_3.pdf.

7. Bonilla R, Herrera R, López M, Lugo M, Rodríguez O, Rodríguez S, Ruiz L, Macías C. Programa de educación en salud oral aplicado a un grupo de maternas. Journal Odontológico Colegial. [Internet] 2008 [25.06.14] Disponible en: http://www.journalodontologicocolegial.com/index.php/joc/article/viewFile/132/104.

8. Louise Lin D, Harrison R, Aleksejuniene J. Can a Prenatal Dental Public Health Program Make a Difference? J Can Dent Assoc 2011; 77:b32. [Internet] 2011 [9.08.12] Disponible en: http://www.jcda.ca/article/b32.

9. Buglar ME, White KM, Robinson NG. The role of self-efficacy in dental patients' brushing and flossing: Testing an extended Health Belief Model. QUT Digital Repository [internet] 2010 [3.09.11] Disponible en: http://eprints.qut. edu.au/.

10. Clarkson JE., Young L, Ramsay CR., Bonner B. C. Bonetti D. How to Influence Patient Oral Hygiene Behavior Effectively. J Dent Res. [Internet] 2009 [10.01.13] Disponible en: http://jdr.sagepub.com/content/88/10/933.

11. Silva de Araujo Figueiredo C., Gonçalves Carvalho Rosalem C., Costa Cantanhede A. L., Abreu Fonseca Thomaz

E. B. and Fontoura Nogueira da Cruz M.C. Systemic alterations and their oral manifestations in pregnant women. J. Obstet. Gynaecol. [internet] 2017 [21.04.17] Disponible en: http://onlinelibrary.wiley.com/doi/10.1111/jog.13150/pdf. 
12. Hwang S, Smith V, McCormick M C, Barfield W. The Association between Maternal Oral Health Experiences and Risk of Preterm Birth in 10 States, Pregnancy Risk Assessment Monitoring System, 2004-2006. Matern Child Health J. [internet] (2012) [21.04.17] Disponible en: http://eds.b.ebscohost.com/eds/pdfviewer/pdfviewer?vid=3\&sid=db3f43130bd9-4e6e-a23c-9bf53db3a700\%40sessionmgr103\&hid=117.

Recibido 20/12/2016

Aceptado 04/07/2017

Correspondencia: Rossana Sotomayor Ortellado sotomayorr12@gmail.com 